\title{
Generator Looseness Fault Diagnosis Based on Time-Frequency Analysis
}

\author{
Ting $\mathrm{Li}^{1, \mathrm{a}}$, Chen Liu ${ }^{1, \mathrm{~b}}$, Chendong Duan ${ }^{1, \mathrm{c}}, \mathrm{Xia} \mathrm{Qi}^{1, \mathrm{~d}}$ \\ ${ }^{1}$ School of electronic \& control engineering, Chang'an University, Xi'an, 710064, China \\ altlx_li@163.com, ${ }^{\mathrm{b}} 821806702 @ q q . c o m,{ }^{\mathrm{c}}$ cdduan@.chd.edu.cn, ${ }^{\mathrm{d}}$ tnndgbt@126.com
}

Key words: Frequency Slice Wavelet Transform; Looseness Fault; Feature Extraction

\begin{abstract}
In order to extract looseness fault feature for a generator set, the paper introduces a new time-frequency analysis method, called frequency slice wavelet transform (FSWT). The time-frequency distribution of signal is firstly processed by the FSWT. Then some interesting regions on the FSWT time-frequency map are selected for further analysis. Based on zoom and refine analysis, fault features are finally extracted and separated. Experiment and engineering application research showed that this method is effective.
\end{abstract}

\section{Introduction}

Looseness fault is a kind of fault in rotating machinery, which sometimes could cause catastrophic accident. When looseness fault appears, the machine behaviors usually are non-stationary and non-linear ${ }^{[1]}$. Some classical methods are not effective under this situation, so new time-frequency analysis methods are applied continuously to expect to discover more efficient features for diagnosis, such as the Short-Time Fourier transform (STFT), the Wigner-Ville distribution (WVD), wavelet transform (WT), and so on ${ }^{[2]}$.But researchers found that these methods have disadvantages in applications. The time-frequency window size of the STFT is fixed, which leads to signal aliasing is relatively serious near the characteristic frequency, so it has the low frequency resolution. The WVD has overlapping interference item in time-frequency plane for a signal with multiple components, so the extraction and interpretation of the fault features is difficult; in the WT, most wavelet functions are not symmetrical, thus during transformation the observed frequency and the time-frequency window center frequency of the wavelet are not conformity ${ }^{[3]}$. In order to obtain more effective features for generator set diagnosis, a new time-frequency processing method, the Frequency Slice Wavelet Transform (FSWT), is introduced in this paper. Vibration signal from the set is firstly dealt with the FSWT to get the time-frequency energy distribution. Then, some special regions are chosen to make further analyses for finding fault features. Experiment and engineering application showed that this method is effective.

\section{Frequency Slice Wavelet Transform}

For any $f(t) \in L^{2}(R)$, suppose $\hat{f}(u)$ is Fourier transformation of $f(t), \hat{p}(u)$ is Fourier transformation of $p(t), \hat{p}^{*}(\omega)$ is conjugate function of frequency slice function (FSF) $\hat{p}(\omega)$. The frequency slice wavelet transform (FSWT) of $f(t)$ is defined as:

$W(t, \omega, \sigma)=\frac{1}{2 \pi} \int_{-\infty}^{+\infty} \hat{f}(u) \hat{p}^{*}\left(\frac{u-\omega}{\sigma}\right) e^{i u t} d u$.

Where $\sigma(\sigma \neq 0)$ is the scale factor which is a constant or a function of $\omega, t$ and $u^{[4]}$.

According to the principle of Morlet wavelet transform, let $\sigma=\frac{\omega}{\kappa}$ to adjust the sensitivity of the transformation frequency or time, where $k(k>0)$ is defined as time-frequency resolution factor and doesn't directly relate to $\omega$ and $u^{[5]}$. Eq. (1) can be changed into

$$
W(t, \omega, \kappa)=\frac{1}{\kappa} \omega e^{i \omega t} \int_{-\infty}^{+\infty} f(\tau) e^{i \omega \tau} p^{*}\left[\frac{\omega(\tau-t)}{\kappa}\right] d \tau .
$$


The inverse transformation of FSWT is defined as:

$$
f(t)=\frac{1}{2 \pi} \int_{-\infty}^{+\infty} \int_{-\infty}^{+\infty} W(\tau, \omega, \kappa) e^{i \omega(t-\tau)} d \tau d \omega .
$$

It's obviously that there is no directly relationship between the reconstructed transform Eq. (3) and the frequency slice function $\hat{p}(\omega)$, only the scale factor $\sigma$ have relation with the reconstruction ${ }^{[6]}$.

\section{Simulation experiment}

A signal $f(t)$ is defined as:

$$
f(t)=\sum_{i=1}^{6} A_{i} \cos \left(2 \pi f_{i} t\right)+n(t)
$$

Where $A_{1}=A_{2}=2, A_{3}=A_{4}=A_{5}=A_{6}=1, f_{1}=200 \mathrm{~Hz}, f_{2}=400 \mathrm{~Hz}$ is the rotation frequency, $\mathrm{f}_{3}=600 \mathrm{~Hz}, \mathrm{f}_{4}=800 \mathrm{~Hz} \mathrm{f}_{5}=1000 \mathrm{~Hz}, \mathrm{f}_{6}=1200 \mathrm{~Hz}, \mathrm{n}(\mathrm{t})$ is white noise with $\mathrm{SNR}=0.5$. The sampling frequency is $f_{s}=5000 \mathrm{~Hz}$, the length of signal is 1024 . The signal is shown in Fig.1.
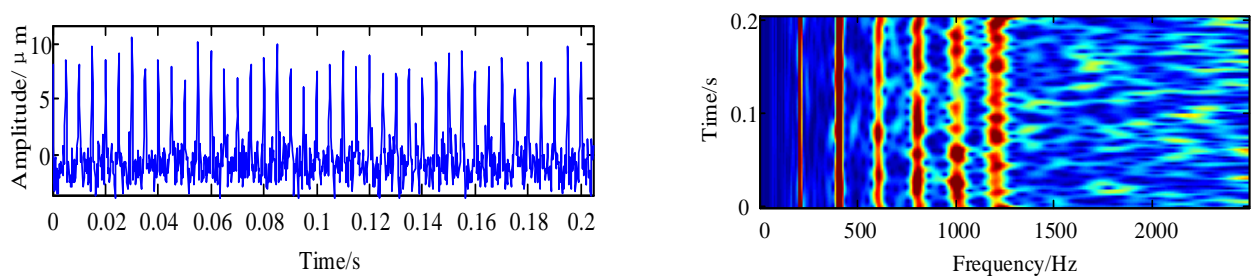

Fig.1 Simulated signal

Fig.2 Time-frequency map of simulated signal

Fig. 2 shows the time-frequency map of $f(t)$ after transforming with FSWT. The component of signal is a series of cosine signal with integer-times frequency of $200 \mathrm{~Hz}$. It's shown that there are several color pieces significantly different from other areas which is the harmonics of $200 \mathrm{~Hz}$ over frequency-band $[0 \mathrm{~Hz}, 1300 \mathrm{~Hz}]$, while frequency-band $[1200 \mathrm{~Hz}, 2500 \mathrm{~Hz}]$ reflects the noise signal.

Fig.3 shows the original signal and reconstructed signal from separated signal over frequency-band $[390 \mathrm{~Hz}, 410 \mathrm{~Hz}]$ through FSWT inverse transformation Eq.(3). The average error between original signal and reconstructed signal is -0.0017 . The cross correlation coefficient between them is 0.9947 and reveals that the information of the original signal is kept well by reconstructed signal.

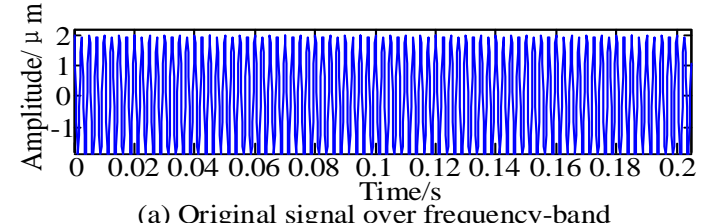

(a) Original signal over frequency-band [390Hz,410Hz]

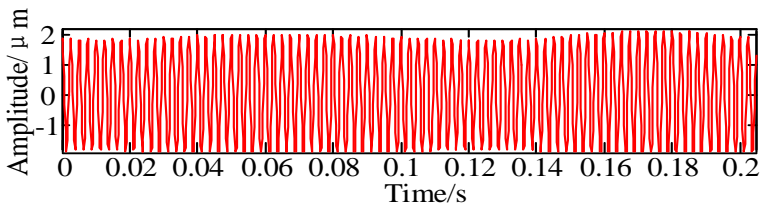

(b) Reconstructed signal of $400 \mathrm{~Hz}$ over frequency-band [390Hz,410Hz]

Fig.3 Original signal and its reconstructed signal

\section{Engineering application}

Fig. 4 shows the structure of generator set, 8 accelerators are mounted on bearing bush to monitor the set operation condition denoted No.1 No.8. After an overhaul, the set started again. The monitoring system found that vibration on Bush No.4 was serious during the set booting up and uploading. It was observed that vibration value on Bush No.4 became greater with the operation speed increased, when the set was under no-load operation, the vibration peak-to-peak value of Bush No.4 is $24.7 \mu \mathrm{m}$ at the speed of $740 \mathrm{r} / \mathrm{min}, 63.2 \mu \mathrm{m}$ at the speed of $3000 \mathrm{r} / \mathrm{min}$. But peak-to-peak values of the other bushes were not greater than $50 \mu \mathrm{m}$ at all. To investigate what the reason caused, we took the vibration signal obtained from Bush No.4 for analysis. 


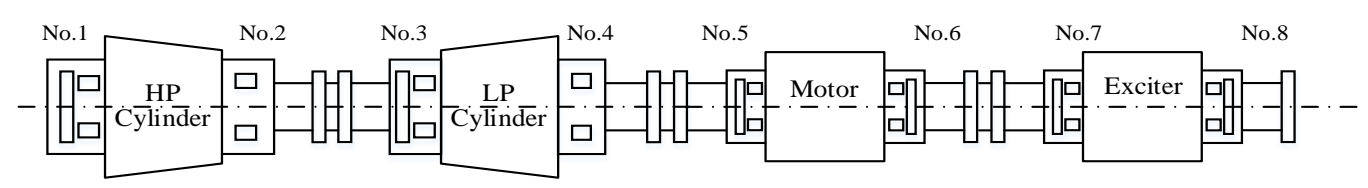

Fig.4Monitoring setting structure

Fig. 5 shows a vibration signal from Bush No.4 when the set uploaded $6 \mathrm{MW}$ load at the speed of $3000 \mathrm{r} / \mathrm{min}$, the length of the signal is 1024, and the sampling frequency is $2000 \mathrm{~Hz}$. Fig. 6 displays its spectrum. It can be seen that the signal amplitude reached a maximum in operation frequency $50 \mathrm{~Hz}$, and there are several harmonic of $50 \mathrm{~Hz}(2 \mathrm{x}$ to $11 \mathrm{x}$ ) from $100 \mathrm{~Hz}$ to $550 \mathrm{~Hz}$ in signal vibration spectrum.

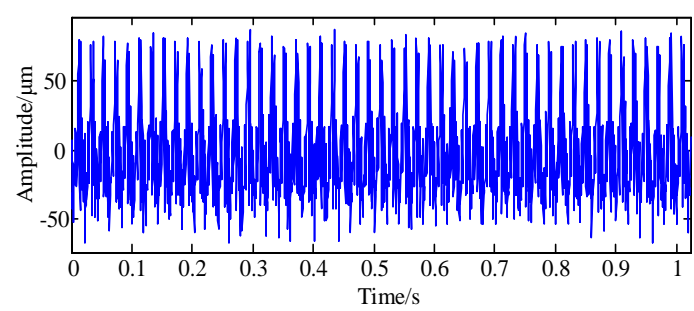

Fig.5 Signal before repair

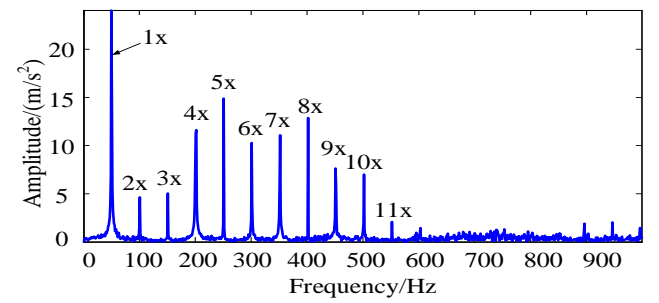

Fig.6 Signal spectrum before repair

In order to get much more detail information, the original signal is decomposed using the FSWT with slice function $p(\omega)=1 /\left(1+w^{2}\right)$. The time-frequency map is displayed in Fig.7. As we observed in Fig.7, the energy is concentrated under the frequency of $600 \mathrm{~Hz}$, it is the same as in Fig.6, these components in low frequency-band under $600 \mathrm{~Hz}$ are the operation frequency and its harmonics. However high frequency components over the frequency $600 \mathrm{~Hz}$ have no significant signatures, they can be thought as high frequency noise produced by the set. As a result, we choose the low frequency-band as slice 1 for further analysis.

Fig.8 shows the zoom map under $600 \mathrm{~Hz}$. In Fig.8 some highlight color bars are distinguished, these color bars demonstrates impact vibration signal characteristics which has short duration time and swing frequency in a wide range. The center frequencies of these bars are the operation frequency $50 \mathrm{~Hz}$ and its harmonics (up to 11 times the operation frequency). These bars' signatures on the time-frequency map reflect the randomness and non-stationary in the vibration signal. Fig.9 displays the rebuilt signal from the band $[200 \mathrm{~Hz}, 500 \mathrm{~Hz}]$ in Fig.8, it obviously is a series of impact responses
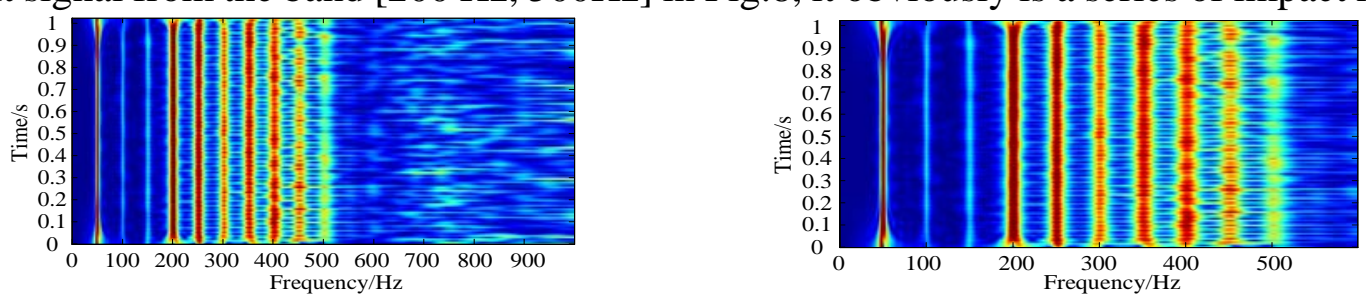

Fig.7 Time-frequency map before repair Fig.8 The zoom time-frequency map of slice 1

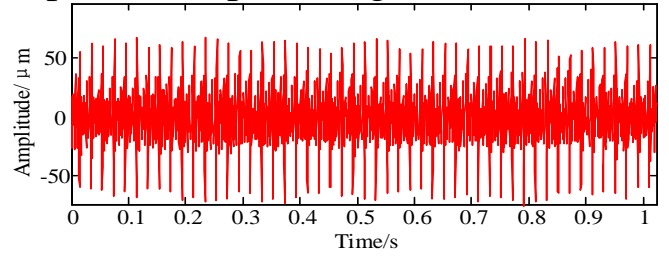

Fig.9 Reconstructed signal in [200,500Hz]

As investigated above, the signal of Bush No. 4 is non-stationary and nonlinear. Because these was no symptoms of rotor imbalance and misalignment fault found after field checking, so we thought that there would be looseness fault in the set.

After repairing, the set started again. When it run at the speed of $3000 \mathrm{r} / \mathrm{min}$ under load 6MW, 20MWand 45MW, vibration peak-to-peak values at Bush No.4 were $45 \mu \mathrm{m}, 40 \mu \mathrm{m}$ and $55 \mu \mathrm{m}$ respectively. The values were not greatly increased yet. The set was in operation. 
Fig.10 shows a signal from Bush No. 4 after repair. Compared with Fig.5, the signal was more symmetry, and the signal amplitude was decrease significantly (see Fig.11). Fig.12 shows its FSWT map. The operation frequency $(50 \mathrm{~Hz})$ energy was decreased extensively, this means that the loose phenomenon was effectively relieved. The reconstructed signal over the band $[200 \mathrm{~Hz}, 500 \mathrm{~Hz}]$ is given as in Fig.13, its amplitude is also decreased prominently.

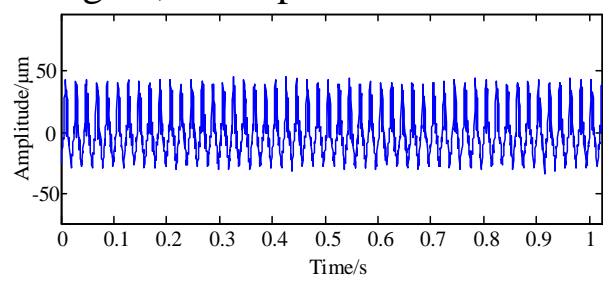

Fig.10 Signal after repair

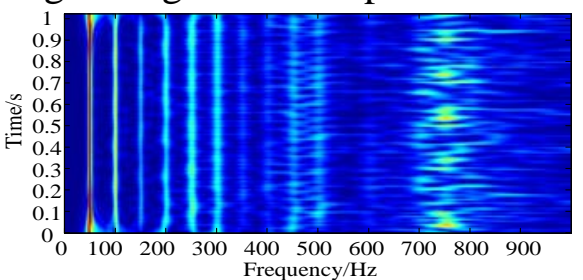

Fig.12 Time-frequency map after repair

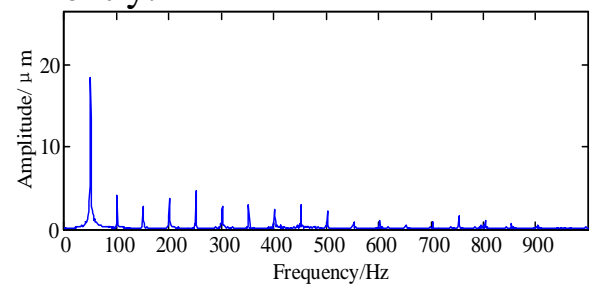

Fig.11 Fourier spectrum after repair

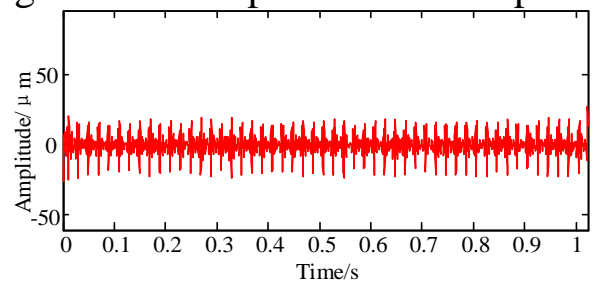

Fig.13 Reconstructed signal after repair

Fig.14 displays instantaneous frequency curves of the signals from No.4 Bush before and after repair. Table 1 lists some indexes of these 2 curves, such as Root Mean Square(RMS), average(AVG), variance (VAR) and maximum(MAX). After repaired, these index values are much more less than that before repaired.
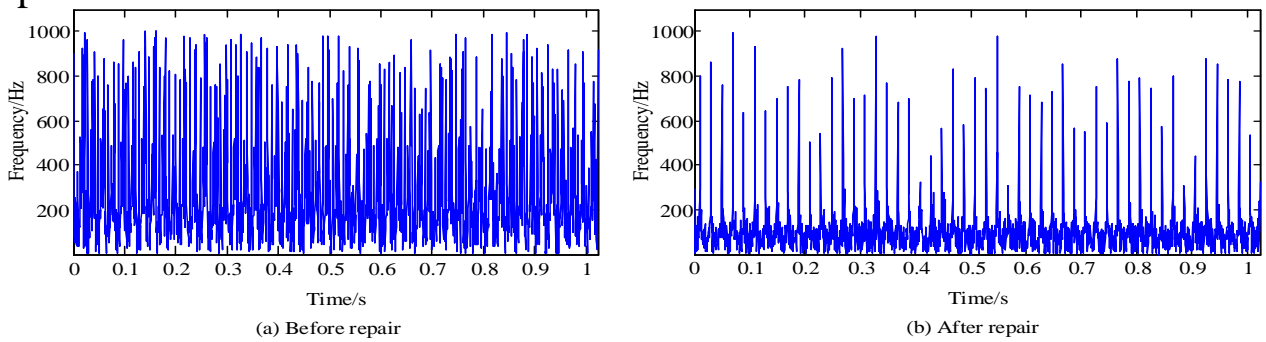

Fig.14 Instantaneous frequency before and after repair

Table 1 Statistical characteristics of slice 1

\begin{tabular}{ccc}
\hline & Before repair & After repair \\
\hline RMS & 304.1372 & 115.1906 \\
AVG & 123.3092 & 50.6612 \\
VAR & 278.0929 & 103.8331 \\
MAX & 996.2605 & 667.6961 \\
\hline
\end{tabular}

\section{Conclusion}

It is difficult to analyze the non-stationary signal when there are faults appearing within a generator. This paper use a new time-frequency feature extraction method of FSWT to extract and separate the fault feature. With the aid of FSWT, the multi-component vibration signal can be segmented and extracted based on its time-frequency energy distribution. Moreover, the fault feature can be separated by FSWT reconstruction so that the fault information which contained in the signal can be used to diagnosis. Simulation experiment and engineering application showed that the FSWT method is more effective and adaptive for fault feature extraction in generator looseness fault diagnosis.

\section{Acknowledgements}

This research were financially supported by the Natural Science Foundation of Shanxi (No.2014JM7246). 


\section{Reference}

[1] Z He, Y Zi, Q Meng. Fault diagnosis principle and application of non-stationary signal in mechanical equipment [M]. Higher Education Press, Beijing (In Chinese,2001: 1-4, 96-105).

[2] M J Dehghani. Comparison of S-transform and Wavelet Transform in Power Quality Analysis. Engineering and Technology (2009).

[3] Z Yan, A Miyamoto, Z Jiang, et al. An overall theoretical description of frequency slice wavelet transform. Mechanical Systems and Signal Processing [J] (2010, 24: 491-507).

[4] Z Yan , A Miyamoto , Z Jiang. Frequency slice algorithm for modal signal separation and damping identification. Computers and Structures[J]. $2011,89: 14-26$.

[5] Z Yan , A Miyamoto , Z Jiang. Frequency slice wavelet transform for transient vibration response analysis[J]. Mechanical Systems and Signal Processing (2009, 23:1474-1489).

[6] Cendong Duan, Qiang Gao. Bearing damage feature extraction method based on time-frequency slice analysis [J]. Journal of Vibration and Shock (In Chinese,2012, 31(5)). 